\title{
Genetic variation of aggrecanase-2 (ADAMTS5) in susceptibility to osteoarthritis
}

\author{
Xindie Zhou ${ }^{1,2 *}$, Lifeng Jiang ${ }^{3 *}$, Yi Zhang ${ }^{2}$, Junjie Zhang ${ }^{2}$, Dong Zhou ${ }^{1}$, Lidong $\mathrm{Wu}^{3}$, \\ Yong Huang ${ }^{2}$ and Nanwei $\mathrm{Xu}^{1}$
}

${ }^{1}$ Department of Orthopedics, The Affiliated Changzhou No. 2 People's Hospital of Nanjing Medical University, Changzhou, China

${ }^{2}$ Department of Orthopedic Trauma, The Affiliated Changzhou No. 2 People's Hospital of Nanjing Medical University, Changzhou, China

${ }^{3}$ Department of Orthopedic Surgery, The Second Affiliated Hospital, Zhejiang University School of Medicine, Hangzhou, China

\begin{abstract}
Aggrecanase-2 (ADAMTS5) gene is responsible for aggrecan degradation that may contribute to cartilage destruction in a mouse osteoarthritis (OA) model. We aimed to investigate the effects of ADAMTS5 gene polymorphisms on OA risk in a Chinese population. A total of $300 \mathrm{OA}$ patients and 300 controls were recruited and their genotypes for ADAMTS5 gene rs226794 and rs2830585 polymorphisms were determined using a custom-by-design 48-Plex single nucleotide polymorphism Scan ${ }^{\mathrm{TM}}$ kit. ADAMTS5-associated genes were identified by co-expression analysis and their functions were investigated by Gene Ontology and Kyoto Encyclopedia of Genes and Genomes analyses. Bioinformatics analysis showed that ADAMTS5 was significantly related to the components, structural constituent, and organization of the extracellular matrix. The rs2830585 polymorphism, but not rs226794 polymorphism, was significantly associated with an increased risk of knee OA. Stratified analysis further confirmed this significant association in patients at age $\geqslant 55$ years. In conclusion, the ADAMTS5 rs 2830585 polymorphism may be involved in the development of knee OA by destroying the extracellular matrix, but this finding should be further confirmed by larger studies.
\end{abstract}

Key words: ADAMTS5; Polymorphism; Osteoarthritis; Co-expression analysis

\section{Introduction}

Osteoarthritis (OA) is the most common form of arthritis and a major socioeconomic burden (1). The incidence of $O A$ increases with aging and is higher among women, especially after 50 years of age (2). OA of the hips and knees tends to cause severe disability requiring surgical intervention (3). Notable risk factors of OA include age, obesity, gender, smoking, genetics, diet, and occupation (4). Identification of OA-associated genes can help identify the biological mechanisms of OA (5).

$\mathrm{OA}$ is characterized by degeneration of articular cartilage and changes in periarticular and subchondral bones (6). The degeneration is attributed primarily to uncontrolled destruction of the extracellular matrix (ECM), including the proteoglycan aggrecan and type II collagens (7). Aggrecanase-mediated aggrecan degradation plays an important role in OA development $(8,9)$. Two cartilage aggrecanases [aggrecanase-1 (ADAMTS4) and aggrecanase-2 (ADAMTS5)] have been identified, which are both very efficient in cleaving soluble aggrecan at the Glu (373)-Ala (374) site (10). A surgical mouse OA model shows that ADAMTS5 ablation can essentially eliminate cartilage erosion and fibrous overgrowth (11). Therefore, ADAMTS5 may play a crucial role in OA development.

The function of ADAMTS5 may be influenced by two non-synonymous single nucleotide polymorphisms (SNPs) by altering the amino acid sequence of the protein. The association of ADAMTS5 gene variants [rs226794 (P692L in exon 7) and rs2380585 (R614H in exon 5)] with OA development has been studied in various populations (12-14), but not in East China. Therefore, the present study was conducted to evaluate the effects of ADAMTS5 gene polymorphisms on OA risk in an East Chinese Han Population.

\section{Material and Methods}

\section{Study subjects}

A total of 300 knee OA patients (test group) and 300 controls (control group) were recruited from the Affiliated Changzhou No. 2 People's Hospital of Nanjing Medical University (China) and the Second Affiliated Hospital

Correspondence: Yong Huang: <m13813585151@163.com> | Nanwei Xu: <xunanwei@sina.com>

*These authors contributed equally to this study.

Received August 24, 2018 | Accepted November 7, 2018 
of Medical College, Zhejiang University (China) between October 2013 and November 2017. Knee OA was diagnosed in accordance with the criteria of the American College of Rheumatology (1987) (15): primary OA with any symptom and radiographic sign of OA according to the Kellgren-Lawrence (K-L) grading system. Patients with post-traumatic or post-septic arthritis, inflammatory arthritis, or malignant or chronic illness were excluded. The 300 controls were selected from patients attending orthopedic clinics of the same hospitals for treatment of trauma at the time of sampling. Any subject with doubtful diagnosis was excluded. The functional or symptomatic status of patients was assessed using Lequesne functional index. Pain was evaluated by the visual analogue scale (VAS), a pain measure scale. Controls were selected from the patients attending the general surgery and orthopedics clinics of the two hospitals at the time of sample collection. A questionnaire was designed to collect from cases and controls the general information [e.g., age, sex, body mass index (BMI)] and clinical data [e.g., erythrocyte sedimentation rate (ESR) and C-reactive protein (CRP)] of OA.

This study was approved by the Institutional Ethics Committees of the two hospitals. Written informed consent was obtained from each subject.

\section{Co-expression analysis and SNP selection}

ADAMTS5 coexpressing genes were identified using co-expressed gene database (COXPRESdb) (http://cox presdb.jp/) and their protein interaction networks were constructed using STRING (https://string-db.org/). The most relevant functions of these genes were identified via Gene Ontology (GO) and Kyoto Encyclopedia of Genes and Genomes (KEGG) analyses.

Linkage data were searched from Ensembl (http:// ensembl.org/index.html) and processed on Haploview (https://www.broadinstitute.org/haploview/haploview).

The tag SNPs were selected on Haploview, in which a threshold of $r^{2}>0.8$ was applied in the pairwise correlation, and SNPs with a minor allele frequency $<10 \%$ were excluded. Functional predictions were performed on SIFT (http://sift.jcvi.org/www/SIFT_dbSNP.html).

\section{DNA extraction and genotyping}

Venous blood (2 $\mathrm{mL}$ each) was sampled in tubes containing ethylenediamine-tetraacetic acid (EDTA) and stored at $-80^{\circ} \mathrm{C}$ before use. Genomic DNA was extracted using a QIAamp DNA blood mini kit (Qiagen, Germany), and the concentration and purity were estimated using NanoDrop (Thermo Electron Corp., USA) at two absorbance wavelengths of 260 and $280 \mathrm{~nm}$. Genotyping was done by matrix-assisted laser desorption/ionization time-of-flight mass spectrometry (MALDI-TOFMS) using a MassARRAY system (Sequenom, USA). Completed genotyping reactions were spotted onto a 384-well spectroCHIP (Sequenom) using a MassARRAY nanodispenser (Sequenom) and analyzed by MALDI-TOFMS. Genotype calling was done in real time with MassARRAY RT 3.1 (Sequenom) and analyzed on MassARRAYTyper 4.0 (Sequenom). For quality control, $10 \%$ of randomly-selected samples were analyzed repeatedly.

\section{Enzyme-linked immunosorbent assay (ELISA)}

Serum ADAMTS5 levels of OA patients were measured using the human ADAMTS5 ELISA kit (Biorbyt, UK), according to the manufacturer's recommendations. Absorbance was read at $450 \mathrm{~nm}$ using a microplate reader (TECAN INFINITF*F50, Switzerland). ADAMTS5 concentration could be determined using the standard curve. The detection range of this kit is $15.6-1000 \mathrm{ng} / \mathrm{mL}$. All samples were determined by the same investigator, who was blind to the clinical situation.

\section{Statistical analysis}

Hardy-Weinberg equilibrium (HWE) was assessed using goodness-of-fit $\chi^{2}$ tests to investigate deviation between observed and expected frequencies among controls. Clinical data were compared between groups by the unpaired Student's $t$-test, while qualitative data were compared by the chi-squared test. All genotypes for the two polymorphisms evaluated were divided and analyzed in three distinct genetic models, according to a study by Clarke et al. (16): 1) genotype distribution model (wild-type vs heterozygous vs mutated); 2) dominant genetic model (wild-type vs heterozygous + mutated), and 3 ) recessive genetic model (mutated vs wild-type vs heterozygous + wild-type). Odd ratios (ORs) and 95\% confidence intervals (Cls) were calculated to evaluate the associations between ADAMTS5 gene polymorphisms and $O A$ risk by logistic regression analyses. All statistical analyses were performed on SAS 9.1.3 (SAS Institute, USA) with the significant level at $\mathrm{P}<0.05$.

\section{Results}

\section{Bioinformatics analysis}

The protein-protein association networks co-expressed with ADAMTS5 are shown in Figure 1. The five genes most related to ADAMTS5 were ADAM metallopeptidase with thrombospondin type 1 motif 1 (ADAMTS1), fibrillin 1 (FBN1), laminin alpha 4 (LAMA4), protocadherin 18 (PCDH18), and decorin (DCN). LAMA4-integrin signaling contributes to clustering in human osteoarthritic chondrocytes, which is a morphological sign of OA (17). Figure 2 shows the GO enrichment results of these genes. Annotation results indicate these genes are correlated with ECM component (CC), ECM structural constituent (MF), and ECM organization (BP) terms and the protein digestion and absorption pathway. ADAMTS5 may be involved in OA development through ECM.

The 9 tagger SNPs screened out on Haploview 4.2 are shown in Figure 3. The relevant parameters were set as follows: HW P-value cutoff $=0.05$; $\min$ genotype $=75 \%$; Max\# Mendel error=1; minimum allele frequency $=0.1$. 


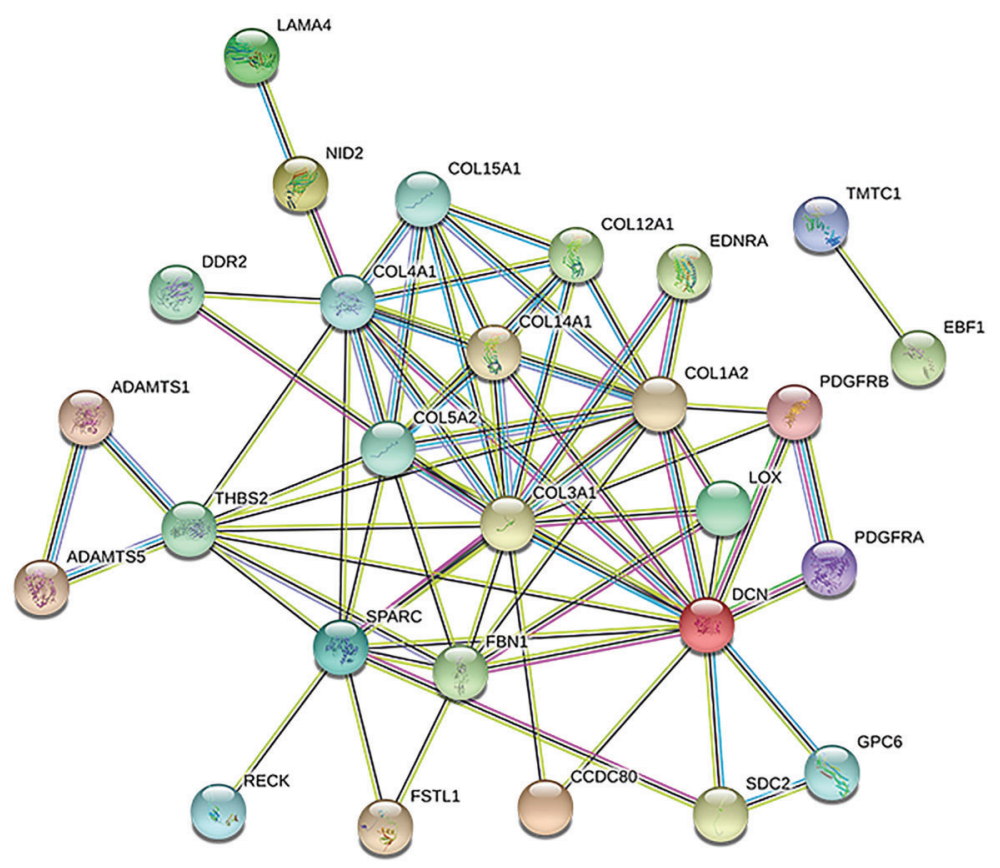

Figure 1. Protein interaction network of ADAMTS5 co-expression gene.

The 9 tagger SNPs included 6 intronic SNPs, 2 missense SNPs, and 1 unknown SNP. SIFT analysis showed the rs226794 polymorphism caused Leu > Pro at amino acid position 692, decreasing the risk of OA. However, rs2830585 was predicted to be deleterious for ADAMTS5 protein function (Table 1).

\section{Characteristics of subjects}

Baseline characteristics are reported in Table 2. The patients were slightly younger and consisted of more females than the controls $(P=0.329 ; 0.719)$. BMI data were significantly different between groups $(P<0.001)$. The majority of OA cases belonged to K-L grade 2 or 3 . We also included several clinical parameters (such as VAS and Lequesne index) to investigate the OA patients' clinical conditions. The observed genotype frequencies of rs226794 in the control group followed the HWE ( $P=0.997)$. The genotype distributions of rs2830585 were not significantly different between groups $(\mathrm{P}=0.961)$.

\section{Associations between ADAMTS5 polymorphisms and OA risk}

Table 3 shows the genotype and allele distributions for ADAMTS5 gene variants in both groups. No significant deviation from HWE was found for two SNPs in the controls ( $P=0.998$ for $r 226794, P=0.961$ for $r 2830585$, respectively). None of the five models showed any significant association between OA risk and rs226794 polymorphism. The TT genotype of rs2830585 polymorphism was significantly associated with a 1.95-fold increased risk of OA compared with the CC genotype (TT vs CC: OR, 1.95; 95\% $\mathrm{Cl}, 1.03-3.71 ; \mathrm{P}=0.041)$. Similarly, the $\mathrm{CT}+\mathrm{TT}$ genotype was significantly associated with an increased risk of OA (CT + TT vs CC: OR, 1.45; 95\% Cl, 1.05-2.00; $\mathrm{P}=0.024)$. After adjusting for gender, age, and BMI, the results were still significant. Furthermore, the rs2830585T allele increased the risk of OA by $39 \%$ compared with the $C$ allele ( $T$ vs C: OR, 1.39; 95\%Cl, 1.07-1.79; $\mathrm{P}=0.013$ ).

Haplotypes were established through the use of two SNPs. The distribution of the haplotype frequency of the two polymorphisms for the OA patients and healthy controls are presented in Table 4. There was no significant difference between $\mathrm{OA}$ patients and controls with regard to haplotype frequencies of $G C, A C$, and $G T(P>0.05)$. Stratified analyses according to sex and age are illustrated in Table 5 . For subjects at age $\geqslant 55$ years, ADAMTS5 rs2830585 polymorphism was significantly associated with an increased risk of OA in the additive model (TT vs CC: OR $3.34 ; 95 \% \mathrm{Cl}, 1.38-8.56 ; \mathrm{P}=0.012)$. Significance was also present in the dominant and recessive models. However, the subgroup analysis of sex showed no significant association. We also investigated the clinical parameters in the different genotypes of rs2830585 polymorphism (Table 6). There was no significant effect on OA risk with regard to BMI, CRP, ESR, VAS, Lequesne index, and K-L grading. Our results indicated that serum ADAMTS5 levels of the TT genotype were higher than that in the CC genotype, but the result was not significant (Figure 4). This indicated that amino 

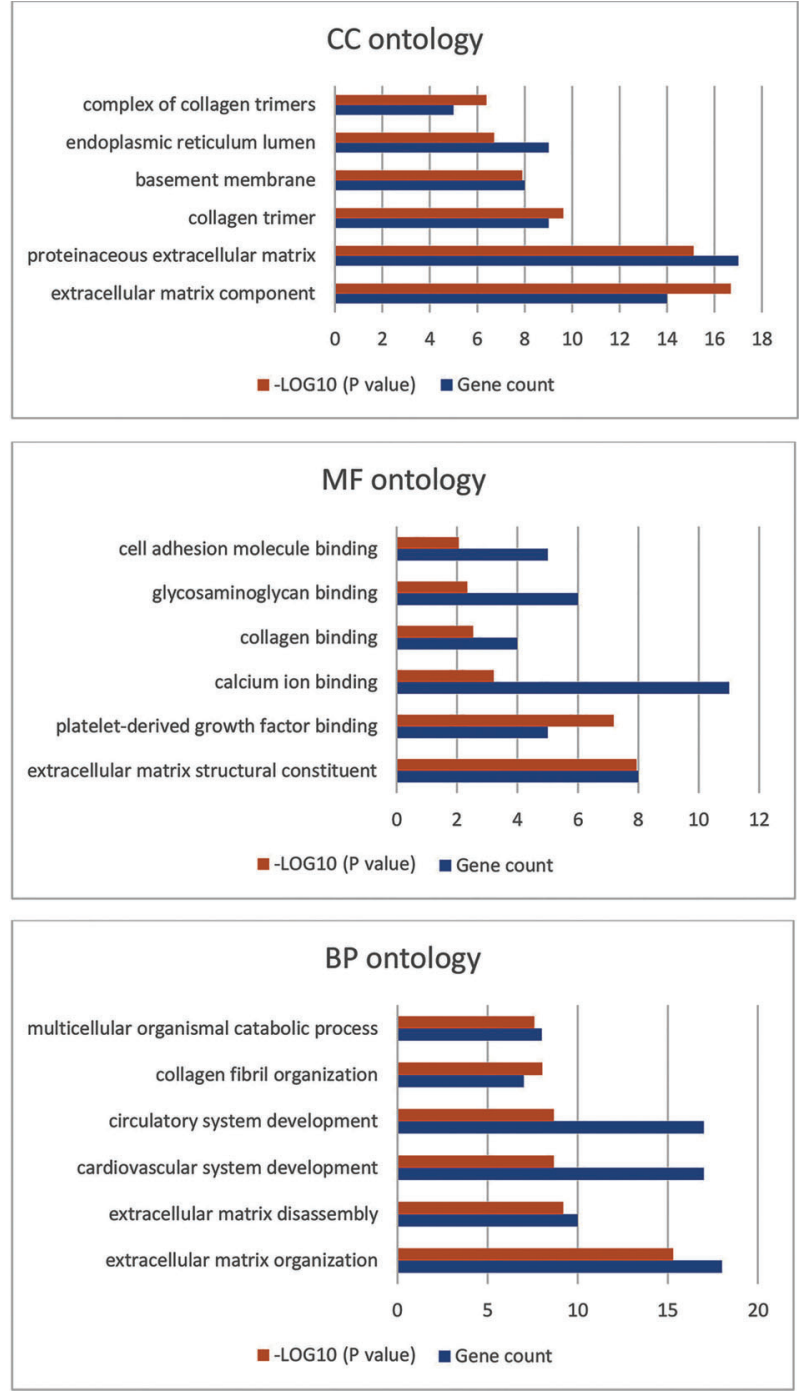

Figure 2. Bar plot of representative Gene Ontology analysis of ADAMTS5 co-expression gene. CC: extracellular matrix (ECM) component; MF: ECM structural constituent; BP: ECM organization.

acid changes (Arg $>$ His) may have no effect on ADAMTS5 protein function and the SIFT prediction may be wrong.

\section{Discussion}

Cartilage consists of a relatively small amount of chondrocytes embedded in abundant ECM, which contains numerous macromolecules, especially collagen fibrils and the large aggregating proteoglycan aggrecan (18). When OA occurs, the degradation of ECM macromolecules surpasses the synthesis, eventually leading to total or partial cartilage erosion (19). ADAMTS5 could cleave the Glu373-Ala374 bond in the inter-globular domain of

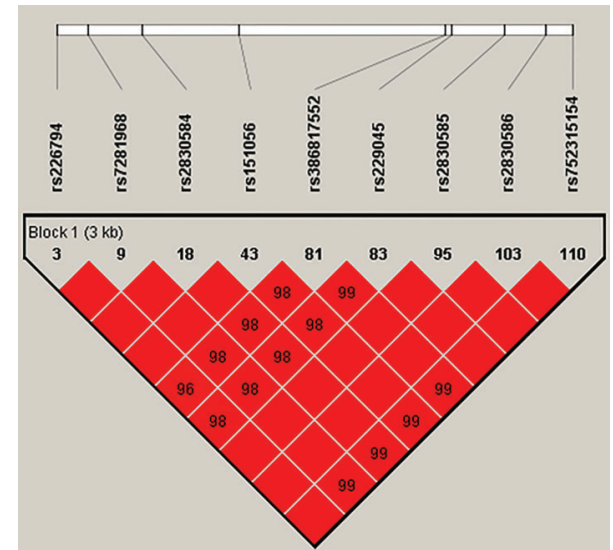

Figure 3. Linkage disequilibrium of the 9 SNPs in ADAMTS5 gene.

aggrecan (20). A murine model of surgically-induced OA shows ADAMTS5 deficiency could diminish aggrecan loss and cartilage erosion (20). Here, we aimed to investigate the function of ADAMTS5 through analyzing the enrichment of its co-expressed genes and found that these genes were significantly associated with the components, structural constituent, and organization of the ECM. These genes were also significantly correlated with platelet-derived growth factor binding, calcium ion binding, and collagen binding, which play important roles in OA development.

Two missense SNPs (rs226794 and rs2830585) in ADAMTS5 were predicted to be deleterious for ADAMTS5 protein function using the SIFT database. The associations between ADAMTS5 gene polymorphisms and OA risk have been investigated in three studies, but with conflicting findings (12-14). Rodriguez-Lopez et al. (12) firstly evaluated the association between ADAMTS5 rs226794 polymorphism and OA risk in 4 Caucasian groups, and found that this polymorphism decreased the risk of knee OA in the Santiago and Thessaly groups (872 cases and 974 controls), but not in the Oxford and Corunna groups. No significant association between ADAMTS5 rs2830585 polymorphism and OA risk was found in the Santiago group (12). Gu et al. (13) reported that the rs2830585 polymorphism, but not the rs226794 polymorphism, was significantly associated with a decreased risk of OA in 420 OA patients compared with 312 controls. The significant association also held true for cervical OA, but not knee, lumbar, or hand OA (13). Canbek et al. (14) revealed that neither ADAMTS5 rs226794 nor rs2830585 polymorphism was linked to susceptibility to knee OA in a Turkish population (80 cases and 95 controls). Here, we validated the association between two ADAMTS5 variants and OA risk in a Chinese population including 300 cases and 300 controls. It was found that rs2830585 polymorphism, but not rs226794 
Table 1. Functional single nucleotide polymorphisms (SNP) selection from the 9 tag SNPs.

\begin{tabular}{|c|c|c|c|c|c|c|c|c|}
\hline SNP & Chr pos (hg38) & Ref/Alt & $\begin{array}{c}\text { dbSNP } \\
\text { func annot }\end{array}$ & $\begin{array}{c}\text { Amino } \\
\text { acid change }\end{array}$ & $\begin{array}{l}\text { Amino } \\
\text { acid }\end{array}$ & $\begin{array}{l}\text { Prediction } \\
\text { (homologs) }\end{array}$ & $\begin{array}{c}\text { Score } \\
\text { (homologs) }\end{array}$ & $\begin{array}{r}\text { Median info } \\
\text { (homologs) }\end{array}$ \\
\hline \multirow[t]{2}{*}{ Rs226794 } & 21:26930036 & $\mathrm{G} / \mathrm{A}$ & missense & L692P & Leu & Damaging & 0.02 & 3.22 \\
\hline & & & & & Pro & Tolerated & 1 & 3.22 \\
\hline Rs7281968 & 21:26930239 & $T / G$ & intronic & & & & & \\
\hline Rs2830584 & $21: 26930587$ & $\mathrm{G} / \mathrm{A}$ & intronic & & & & & \\
\hline Rs151056 & 21:26931204 & $G / A$ & intronic & & & & & \\
\hline Rs386817552 & $21: 26932519$ & $\mathrm{~N} / \mathrm{A}$ & N/A & & & & & \\
\hline Rs229045 & $21: 26932555$ & $\mathrm{G} / \mathrm{A}$ & intronic & & & & & \\
\hline \multirow[t]{2}{*}{ Rs2830585 } & 21:26932893 & $\mathrm{C} / \mathrm{T}$ & missense & $\mathrm{R} 614 \mathrm{H}$ & Arg & Tolerated & 1 & 3.21 \\
\hline & & & & & His & Damaging & 0 & 3.21 \\
\hline Rs2830586 & $21: 26931158$ & $T / G$ & intronic & & & & & \\
\hline Rs752315154 & 21:26933328 & $\mathrm{T} / \mathrm{A}$ & intronic & & & & & \\
\hline
\end{tabular}

Chr pos: chromosome position.

Table 2. Patient demographics and risk factors for knee osteoarthritis.

\begin{tabular}{lccc}
\hline Variable & Cases $(\mathrm{n}=300)$ & Controls $(\mathrm{n}=300)$ & $\mathrm{P}$ \\
\hline Age (years) & $58.2 \pm 9.1$ & $59.0 \pm 9.2$ & 0.329 \\
Sex & & & \\
$\quad$ Male & $85(28.3 \%)$ & $89(29.7 \%)$ & 0.719 \\
$\quad$ Female & $215(71.7 \%)$ & $211(70.3 \%)$ & \\
Body mass index & $26.5 \pm 3.3$ & $24.1 \pm 3.5$ & $<0.001$ \\
CRP, mg/L & $24.8 \pm 14.1$ & & \\
ESR, mm/h & $21.4 \pm 12.6$ & & \\
VAS & $8.1 \pm 2.7$ & & \\
Lequesne index & $14.2 \pm 2.5$ & & \\
Kellgren-Lawrence grading & & & \\
1 & $18(6.1 \%)$ & & \\
2 & $128(42.6 \%)$ & & \\
3 & $98(32.7 \%)$ & & \\
4 & $56(18.6 \%)$ & & \\
\hline
\end{tabular}

Data are reported as number and percentage or mean $\pm S D$ (t-test or chi-squared) test) CRP: C-reactive protein; ESR: erythrocyte sedimentation rate; VAS: visual analogue scale.

polymorphism, conferred susceptibility to knee OA. This discrepancy may be attributed to sample sizes and ethnicitydependent effects. Sample sizes in three other studies were relatively small, and thus their findings may be underpowered. The A allele frequency of Asians was higher than that of Caucasians (0.47 vs 0.09). We hypothesize that genetic heterogeneity, clinical heterogeneity, different genotyping methods, and random errors may also be potential reasons for different findings between Asians and Caucasians.

There is no significant association between ADAMTS5 rs226794 polymorphism and OA risk in any single population (Santiago, Thessaly, Oxford, Corunna, China, and Turkey) (12-14). Rodriguez-Lopez et al. (12) found that this polymorphism decreased the risk of knee OA when combined with Santiago and Thessaly. A possible explanation is that the associations among SNPs in genes associated with OA were greatly affected by the number of participants in the study.

Several studies revealed that ADAMTS5 rs2830585 polymorphism was not linked to susceptibility to OA among Caucasians $(12,14)$. Two Chinese studies evaluated the association between this SNP and OA risk, but with opposite results. The present study shows the $A$ allele of rs2830585 polymorphism increased the risk of $\mathrm{OA}$, while $\mathrm{Gu}$ et al. (13) found it decreased the risk of OA. We hypothesize that environmental factors (e.g., geographic location) impact the genotype distribution of rs2830585 polymorphism. Moreover, eating habits vary geographically in China, which may also explain the above findings. Notably, our study is the first to find a 
Table 3. Logistic regression analysis of associations between ADAMTS5 gene polymorphisms and risk of osteoarthritis.

\begin{tabular}{|c|c|c|c|c|c|c|c|c|c|}
\hline \multirow[t]{2}{*}{ Genotype } & \multicolumn{2}{|c|}{ Cases $^{a}(n=300)$} & \multicolumn{2}{|c|}{ Controls $^{a}(n=300)$} & \multirow{2}{*}{$\begin{array}{c}\text { Hardy-Weinberg } \\
\text { equilibrium }\end{array}$} & \multirow[t]{2}{*}{ OR $(95 \% \mathrm{Cl})$} & \multirow[t]{2}{*}{$\mathrm{P}$} & \multirow{2}{*}{$\begin{array}{l}\text { Adjusted OR } \\
(95 \% \mathrm{Cl})^{\mathrm{b}}\end{array}$} & \multirow[t]{2}{*}{$\mathrm{P}$} \\
\hline & $\mathrm{n}$ & $\%$ & $\mathrm{n}$ & $\%$ & & & & & \\
\hline Rs226794 G/A & & & & & 0.998 & & & & \\
\hline GG & 203 & 67.7 & 182 & 60.7 & & 1.00 & & & \\
\hline GA & 86 & 28.7 & 101 & 33.7 & & $0.76(0.54,1.08)$ & 0.131 & $0.78(0.54,1.13)$ & 0.191 \\
\hline AA & 9 & 3.0 & 14 & 4.7 & & $0.58(0.24,1.36)$ & 0.210 & $0.64(0.26,1.59)$ & 0.335 \\
\hline $\mathrm{GA}+\mathrm{AA}$ & 95 & 31.7 & 115 & 38.4 & & $0.74(0.53,1.04)$ & 0.081 & $0.76(0.53,1.09)$ & 0.140 \\
\hline $\mathrm{GG}+\mathrm{GA}$ & 289 & 96.4 & 283 & 94.4 & & 1.00 & & & \\
\hline $\mathrm{AA}$ & 9 & 3.0 & 14 & 4.7 & & $0.63(0.27,1.48)$ & 0.288 & $0.69(0.28,1.71)$ & 0.426 \\
\hline G allele & 492 & 82.0 & 465 & 77.5 & & 1.00 & & & \\
\hline A allele & 104 & 17.3 & 129 & 21.5 & & $0.76(0.57,1.02)$ & 0.064 & & \\
\hline Rs2830585 C/T & & & & & 0.961 & & & & \\
\hline $\mathrm{CC}$ & 145 & 48.3 & 172 & 57.3 & & 1.00 & & & \\
\hline CT & 126 & 42.0 & 109 & 36.3 & & $1.37(0.98,1.92)$ & 0.068 & $1.41(0.99,2.02)$ & 0.060 \\
\hline TT & 28 & 9.3 & 17 & 5.7 & & $1.95(1.03,3.71)$ & 0.041 & $2.07(1.05,4.10)$ & 0.037 \\
\hline $\mathrm{CT}+\mathrm{TT}$ & 154 & 51.3 & 126 & 42.0 & & $1.45(1.05,2.00)$ & 0.024 & $1.50(1.06,2.11)$ & 0.021 \\
\hline $\mathrm{CC}+\mathrm{CT}$ & 273 & 90.3 & 281 & 93.6 & & 1.00 & & & \\
\hline TT & 28 & 9.3 & 17 & 5.7 & & $1.71(0.91,3.19)$ & 0.093 & $1.79(0.92,3.47)$ & 0.087 \\
\hline C allele & 416 & 69.3 & 453 & 75.5 & & 1.00 & & & \\
\hline T allele & 182 & 30.3 & 143 & 23.8 & & $1.39(1.07,1.79)$ & 0.013 & & \\
\hline
\end{tabular}

${ }^{a}$ Genotyping was successful in 298 cases and 297 controls for rs226794, and 299 cases and 298 controls for rs2830585. ${ }^{\text {b Adjusted for }}$ sex, age, and body mass index. Bold values are statistically significant $(P<0.05)$.

Table 4. Estimated haplotype number and relative frequencies for the two ADAMTS5 variants (rs226794 and rs2830585).

\begin{tabular}{lrccc}
\hline Haplotype & OA & Control & OR $(95 \% \mathrm{Cl})$ & $\mathrm{P}$ \\
\hline GC & $263(0.438)$ & $265(0.442)$ & $0.99(0.81,1.22)$ & 0.942 \\
AC & $82(0.137)$ & $109(0.182)$ & $0.75(0.55,1.02)$ & 0.070 \\
GT & $149(0.248)$ & $118(0.197)$ & $1.26(0.97,1.65)$ & 0.087 \\
\hline
\end{tabular}

OA: osteoarthritis. Chi-squared test.

Table 5. Stratified analyses between ADAMTS5 gene polymorphisms and the risk of osteoarthritis.

\begin{tabular}{|c|c|c|c|c|c|c|}
\hline \multirow[t]{2}{*}{ Variable } & \multicolumn{3}{|c|}{ ADAMTS5 rs2830585 (case/control) } & \multirow{2}{*}{$\begin{array}{c}\text { TT vs CC OR } \\
(95 \% \mathrm{Cl}) ; \mathrm{P} \text { value }\end{array}$} & \multirow{2}{*}{$\begin{array}{c}\text { TT + CT vs CC OR } \\
(95 \% \text { Cl); P value }\end{array}$} & \multirow{2}{*}{$\begin{array}{l}\text { TT vs CT + CC OR } \\
\text { (95\%Cl); P value }\end{array}$} \\
\hline & $\mathrm{CC}$ & $\mathrm{TC}$ & TT & & & \\
\hline \multicolumn{7}{|l|}{ Sex } \\
\hline Male & $49 / 47$ & $30 / 39$ & $5 / 3$ & 2.20 (0.78-6.23); 0.138 & $1.21(0.66-2.21) ; 0.542$ & $2.14(0.80-5.72) ; 0.131$ \\
\hline Female & $96 / 125$ & $96 / 70$ & $23 / 14$ & $1.81(0.76-4.32) ; 0.182$ & 1.60 (0.95-2.69); 0.078 & 2.61 (1.08-6.33); 0.415 \\
\hline \multicolumn{7}{|l|}{ Age (years) } \\
\hline$<55$ & $46 / 52$ & $48 / 35$ & $10 / 5$ & 1.07 (0.41-2.82); 0.890 & 1.09 (0.61-1.94); 0.766 & $1.02(0.41-2.51) ; 0.972$ \\
\hline$\geqslant 55$ & $99 / 120$ & $78 / 74$ & $18 / 12$ & 3.34 (1.30-8.56); 0.012 & $1.80(1.05-3.11) ; 0.034$ & 2.61 (1.08-6.33); 0.034 \\
\hline
\end{tabular}

Bold values are statistically significant $(P<0.05$, chi-squared test).

significant association between ADAMTS5 rs2830585 polymorphism and the risk of knee OA. Gu et al. (13) found TT genotype of rs2830585 decreased the risk of cervical OA, which may be partly attributed to the inherent heterogeneity of disease progression in different types of OA. The rs2830585 polymorphism causes Arg $>$ His at 
Table 6. Comparison of studied data according to ADAMTS5 genotypes in all osteoarthritis (OA) cases.

\begin{tabular}{|c|c|c|c|c|}
\hline \multirow[t]{2}{*}{ ADAMTS5 rs2830585 } & \multicolumn{3}{|c|}{ OA $(n=300)$} & \multirow[t]{2}{*}{$\mathrm{P}$} \\
\hline & $C C(n=145)$ & $A G(n=126)$ & GG $(n=28)$ & \\
\hline BMI $\left(\mathrm{kg} / \mathrm{m}^{2}\right.$, mean $\left.\pm \mathrm{SD}\right)$ & $26.65 \pm 3.15$ & $26.40 \pm 3.57$ & $26.56 \pm 3.06$ & 0.823 \\
\hline $\mathrm{ESR}(\mathrm{mm} / \mathrm{h}$, mean $\pm \mathrm{SD})$ & $21.26 \pm 11.04$ & $21.51 \pm 14.23$ & $21.76 \pm 13.11$ & 0.725 \\
\hline $\mathrm{CRP}(\mathrm{mg} / \mathrm{L}$, mean $\pm \mathrm{SD})$ & $25.73 \pm 14.45$ & $24.45 \pm 14.05$ & $22.63 \pm 11.56$ & 0.504 \\
\hline VAS $($ mean $\pm S D)$ & $8.12 \pm 2.60$ & $8.04 \pm 2.60$ & $8.50 \pm 3.29$ & 0.711 \\
\hline Lequesne index (mean $\pm S D$ ) & $13.96 \pm 2.49$ & $14.37 \pm 2.54$ & $14.43 \pm 2.51$ & 0.342 \\
\hline K-L grading (III + IV / I + II, n (\%)) & $75(51.7 \%) / 70(48.3 \%)$ & $65(51.6 \%) / 61(48.4 \%)$ & $13(46.4 \%) / 15(53.6 \%)$ & 0.870 \\
\hline
\end{tabular}

BMI: body mass index; ESR: erythrocyte sedimentation rate; CRP: C-reactive protein; VAS: visual analogue scale; K-L: KellgrenLawrence. The $t$-test and chi-squared test were used for statistical analyses. Genotyping was successful in 299 OA patients for rs2830585.

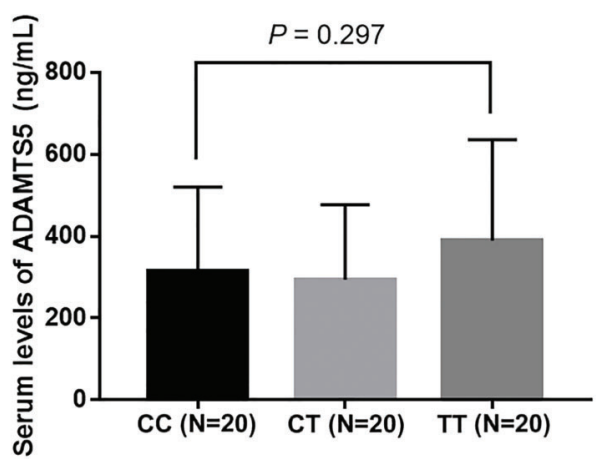

Figure 4. Histogram of serum ADAMTS5 levels in patients with $\mathrm{OA}$. Data are reported as means \pm SD ( $t$-test).

amino acid position 614 when the nucleotide changed from $\mathrm{C}$ to T. Paired amino acid converting enzyme 4 (PACE4) was identified as a proprotein convertase responsible for activation of aggrecanases (ADAMTS4 and ADAMTS5) (21). We hypothesized that amino acid change alters the spatial structure, making it easier to cleave by PACE4, which in turn increases the expression of ADAMTS5. The activation of ADAMTS5 conferred susceptibility to OA. Considering that the rs2830585 polymorphism may

\section{References}

1. Beyer C, Zampetaki A, Lin NY, Kleyer A, Perricone C, lagnocco $A$, et al. Signature of circulating microRNAs in osteoarthritis. Ann Rheum Dis 2015; 74: e18, doi: 10.1136/ annrheumdis-2013-204698.

2. Morgan AP, Knight DC, Tilney NL, Lazarus JM. Femoral triangle sepsis in dialysis patients: frequency, management, and outcome. Ann Surg 1980; 191: 460-464, doi: 10.1097/ 00000658-198004000-00012.

3. Litwic A, Edwards MH, Dennison EM, Cooper C. Epidemiology and burden of osteoarthritis. Br Med Bull 2013; 105: 185-199, doi: 10.1093/bmb/lds038.

4. Leung GJ, Rainsford KD, Kean WF. Osteoarthritis of the hand I: aetiology and pathogenesis, risk factors, investigation contribute to developmental issues that later manifest as OA, we will conduct long-term follow-up of different genotypes in the OA group and control group to observe the effect of genetic factors on OA pathogenesis.

Several possible limitations need to be addressed. First, the population of this hospital-based study may not be representative of the general population. Second, the sample size, which is not particularly small, is still insufficient to obtain significant results. Third, our results may be affected by confounding factors such as alcohol drinking and smoking. Fourth, the two variants genotyped here do not completely cover the whole ADAMTS5 gene. Fifth, this was a retrospective study and we could not provide follow-up data.

In conclusion, ADAMTS5 affected the OA development through ECM, and the ADAMTS5 gene rs2830585 polymorphism was a genetic contributor to risk of knee $\mathrm{OA}$. These findings may contribute to biomarker development for early detection and risk stratification, but should be confirmed by further studies in larger populations.

\section{Acknowledgments}

This study was supported in part by National Natural Science Foundation of China (81702179).

and diagnosis. J Pharm Pharmacol 2014; 66: 339-346, doi: $10.1111 / j p h p .12196$.

5. Hochberg MC, Yerges-Armstrong L, Yau M, Mitchell BD. Genetic epidemiology of osteoarthritis: recent developments and future directions. Curr Opin Rheumatol 2013; 25: 192197, doi: 10.1097/BOR.0b013e32835cfb8e.

6. Goldring MB, Goldring SR. Osteoarthritis. J Cell Physiol 2007; 213: 626-634, doi: 10.1002/jcp.21258.

7. Itoh Y. Metalloproteinases: potential therapeutic targets for rheumatoid arthritis. Endocr Metab Immune Disord Drug Targets 2015; 15: 216-222, doi: 10.2174/1871530315666150316122335.

8. Yatabe T, Mochizuki S, Takizawa M, Chijiiwa M, Okada A, Kimura T, et al. Hyaluronan inhibits expression of ADAMTS4 
(aggrecanase-1) in human osteoarthritic chondrocytes. Ann Rheum Dis 2009; 68: 1051-1058, doi: 10.1136/ard.2007. 086884.

9. Cheung KS, Hashimoto K, Yamada N, Roach HI. Expression of ADAMTS-4 by chondrocytes in the surface zone of human osteoarthritic cartilage is regulated by epigenetic DNA de-methylation. Rheumatol Int 2009; 29: 525-534, doi: 10.1007/s00296-008-0744-z.

10. Tortorella MD, Malfait AM, Deccico C, Arner E. The role of ADAM-TS4 (aggrecanase-1) and ADAM-TS5 (aggrecanase2) in a model of cartilage degradation. Osteoarthritis Cartilage 2001; 9: 539-552, doi: 10.1053/joca.2001.0427.

11. Li J, Anemaet W, Diaz MA, Buchanan S, Tortorella M, Malfait $\mathrm{AM}$, et al. Knockout of ADAMTS5 does not eliminate cartilage aggrecanase activity but abrogates joint fibrosis and promotes cartilage aggrecan deposition in murine osteoarthritis models. J Orthop Res 2011; 29: 516-522, doi: 10.1002/jor.21215.

12. Rodriguez-Lopez J, Mustafa Z, Pombo-Suarez M, Malizos $\mathrm{KN}$, Rego I, Blanco FJ, et al. Genetic variation including nonsynonymous polymorphisms of a major aggrecanase, ADAMTS-5, in susceptibility to osteoarthritis. Arthritis Rheum 2008; 58: 435-441, doi: 10.1002/art.23201.

13. Gu J, Rong J, Guan F, Jiang L, Zhang T, Tao S, et al. Association of ADAMTS5 gene polymorphisms with osteoarthritis in Chinese Han population: a community-based case-control study. Rheumatol Int 2013; 33: 2893-2897, doi: 10.1007/ s00296-012-2506-1.

14. Canbek U, Imerci A, Kara M, Akgun U, Canbek TD, Aydogan $\mathrm{NH}$. Polymorphisms in ADAMTS4 and ADAMTS5 are not linked to susceptibility to knee osteoarthritis in the Turkish population. Genet Mol Res 2016; 15.

15. Silman AJ. The 1987 revised American Rheumatism Association criteria for rheumatoid arthritis. $\mathrm{Br} \mathrm{J}$ Rheumatol 1988; 27: 341-343, doi: 10.1093/rheumatology/27.5.341.

16. Clarke GM, Anderson CA, Pettersson FH, Cardon LR, Morris $\mathrm{AP}$, Zondervan KT. Basic statistical analysis in genetic casecontrol studies. Nat Protoc 2011; 6: 121-133, doi: 10.1038/ nprot.2010.182.

17. Moazedi-Fuerst FC, Gruber G, Stradner MH, Guidolin D, Jones JC, Bodo K, et al. Effect of Laminin-A4 inhibition on cluster formation of human osteoarthritic chondrocytes. J Orthop Res 2016; 34: 419-426, doi: 10.1002/jor.23036.

18. Caterson B, Flannery CR, Hughes CE, Little CB. Mechanisms involved in cartilage proteoglycan catabolism. Matrix Biol 2000; 19: 333-344, doi: 10.1016/S0945-053X(00) 00078-0.

19. Nagase $\mathrm{H}$, Kashiwagi M. Aggrecanases and cartilage matrix degradation. Arthritis Res Ther 2003; 5: 94-103, doi: 10.1186/ ar630.

20. Little CB, Meeker CT, Golub SB, Lawlor KE, Farmer PJ, Smith $\mathrm{SM}$, et al. Blocking aggrecanase cleavage in the aggrecan interglobular domain abrogates cartilage erosion and promotes cartilage repair. J Clin Invest 2007; 117: 1627-1636, doi: 10.1172/JCl30765.

21. Malfait AM, Arner EC, Song RH, Alston JT, Markosyan S, Staten N, et al. Proprotein convertase activation of aggrecanases in cartilage in situ. Arch Biochem Biophys 2008; 478: 43-51, doi: 10.1016/j.abb.2008.07.012. 